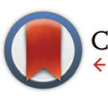

CrossMark \& click for updates

Cite this: Food Funct., 2016, 7, 2197

\title{
Acute effects of chlorogenic acids on endothelial function and blood pressure in healthy men and women
}

\author{
Natalie C. Ward, ${ }^{\mathrm{a}, \mathrm{b}}$ Jonathan M. Hodgson, ${ }^{a}$ Richard J. Woodman, ${ }^{\mathrm{C}}$ \\ Diane Zimmermann, ${ }^{d}$ Laure Poquet, ${ }^{d}$ Antoine Leveques, ${ }^{d}$ Lucas Actis-Goretta, ${ }^{d}$ \\ Ian B. Puddey and Kevin D. Croft*a
}

\begin{abstract}
Coffee is a rich source of polyphenols, primarily chlorogenic acids (CGA). Certain polyphenols and polyphenol-rich foods and beverages have been shown to improve endothelial function and lower blood pressure (BP). The aim of the present study was to investigate the acute effect of two doses of CGA (5CGA) on endothelial function and BP. In a cross-over study, 16 healthy men and women received: (i) $0 \mathrm{mg}$ purified 5-CGA (control group); (ii) $450 \mathrm{mg}$ purified 5-CGA; (iii) $900 \mathrm{mg}$ purified 5-CGA; and (iv) $200 \mathrm{mg}$ purified (-)-epicatechin (positive control) in random order one week apart. Peak and continuous mean (60 to 240 s post ischaemia) flow-mediated dilation (FMD) was measured at baseline, $1 \mathrm{~h}$ and $4 \mathrm{~h}$. BP was measured at baseline and every $30 \mathrm{~min}$ to $4 \mathrm{~h}$. Plasma CGA and epicatechin levels were significantly increased at both $1 \mathrm{~h}$ and $4 \mathrm{~h}$ post their respective treatments. Peak FMD was not significantly altered by either dose of 5-CGA or the epicatechin, relative to control ( $p>0.05)$. Relative to control, effects on continuous mean FMD response following $450 \mathrm{mg} 5$-CGA and $900 \mathrm{mg}$ of 5 -CGA $(0.47 \pm 0.16 \%, p=0.016$ and $0.65 \pm 0.16 \%, p<0.001$, respectively) at $1 \mathrm{~h}$ and $(0.18 \pm 0.17 \%, p=0.99$ and $0.44 \pm 0.16 \%, p<0.05$, respectively) at $4 \mathrm{~h}$. There was no significant effect of any of the treatments on BP. In conclusion, the present study has found no significant effect of 5-CGA, at 450 and $900 \mathrm{mg}$, on peak FMD response. However, there were significant improvements in mean post-ischaemic FMD response, particularly at the $1 \mathrm{~h}$ time point in this group of healthy individuals.
\end{abstract}

Received 25th February 2016, Accepted 15th April 2016

DOI: $10.1039 / c 6 f o 00248 j$

www.rsc.org/foodfunction evidence that certain polyphenols can improve endothelial function and lower blood pressure (BP) through effects on NO metabolism. ${ }^{2}$

Coffee is one of the most widely consumed beverages in the world and represents an important dietary source of polyphenolic compounds. The major polyphenolic compounds found in coffee are chlorogenic acids (CGA), which represents $\sim 86 \%$ of total polyphenol content. Coffee drinkers can have a daily intake of $0.5-1 \mathrm{~g}$ per day of CGA, while abstainers typically consume $<100 \mathrm{mg}$ per day. ${ }^{3}$ While the predominant isomer found in coffee is 5-CGA, the actual amount and composition of coffee polyphenols can be affected by brewing methods. ${ }^{4}$ Epidemiological studies, and meta-analysis derived from these studies, have suggested that moderate coffee consumption (3-4 cups per day) is associated with beneficial effects on a range of chronic diseases, including CVD and type 2 diabetes. $^{5-7}$

Several other plant-based polyphenols have been shown to have beneficial effects on NO status and endothelial function. Loke et al. demonstrated that pure quercetin and epicatechin, polyphenols found in fruits, cocoa and tea, significantly

\footnotetext{
${ }^{a}$ School of Medicine \& Pharmacology, University of Western Australia, Perth, Australia. E-mail: kevin.croft@uwa.edu.au; Fax: +61 89224 0246; Tel: +61892240275

${ }^{b}$ School of Biomedical Sciences \& Curtin Health Innovation Research Institute, Curtin University, Perth, Australia

${ }^{c}$ School of Medicine, Flinders University, Adelaide, Australia

${ }^{d}$ Nestle Research Centre, Lausanne, Switzerland
} 
increased NO status $2 \mathrm{~h}$ after ingestion. ${ }^{8}$ Schroeter et al. observed beneficial effects on endothelial function following ingestion of pure epicatechin. ${ }^{9}$ Studies looking specifically at CGA are limited. A recent acute study demonstrated a reduction in BP $1 \mathrm{~h}$ post ingestion of $400 \mathrm{mg}$ of CGA (3-CQA, Sigma Aldrich). There were no significant effects on endothelial function or NO production. ${ }^{10}$

The timing of the measurements, along with the dose of polyphenols given are important considerations. Following ingestion of CGA, peak circulating concentrations of CGA are reached at approximately 1 hour, and CGA metabolites begin to appear in the circulation from approximately 3-4 hours. ${ }^{11}$ With this in mind, the aim of the present study was to investigate the effect of a $450 \mathrm{mg}$ and a $900 \mathrm{mg}$ dose 5-CGA on endothelial function and BP. The doses were chosen to represent the CGAs present in 4 and 8 cups (approx. $111 \mathrm{mg}$ per cup) of coffee respectively. ${ }^{12}$ Endothelial function measurements were taken at baseline and $1 \mathrm{~h}$ and $4 \mathrm{~h}$ post-treatment to coincide with peak plasma concentrations. ${ }^{11} \mathrm{BP}$ was measured at baseline and then every $30 \mathrm{~min}$ post-treatment. Participants were selected based on an impaired endothelial function (a screening peak flow-mediated dilatation (FMD) response between $3-8 \%$ ), but were otherwise healthy. The primary outcome of this study was peak FMD response. The major secondary outcomes were the continuous mean FMD response between 60 to 240 s post-ischaemia, which represents a continuous vascular dilatory response to ischaemia, and clinic BP.

\section{Methods}

\section{Study design}

This was a double-blind, randomised, placebo controlled cross-over trial. Sixteen healthy men $(n=6)$ and women $(n=$ 10) were recruited to the School of Medicine and Pharmacology, Research Studies Unit (Fig. 1). All participants were aged between 18 and 70 years of age and were regular consumers of coffee. Following an informed consent, participants underwent assessment of their flow-mediated dilatation (FMD). Those with an FMD between 3 and $8 \%$ at screening were included into the study. Additional exclusion criteria included; smokers, known food allergies, systolic BP $<100$ or $>160 \mathrm{mmHg}$ and diastolic $\mathrm{BP}<50$ or $>100 \mathrm{mmHg}$, pregnant or lactating women, use of antihypertensive medication, elevated cholesterol or use of cholesterol-lowering medication, recent significant weight loss or gain ( $>6 \%$ of body weight), BMI outside $18-35 \mathrm{~kg} \mathrm{~m}^{-2}$ range, or presence of major chronic illness.

All participants were advised not to consume more than 2 pieces of fruit, red wine or dark chocolate in the $24 \mathrm{~h}$ period prior to their study visit. Tea and coffee consumption was not permitted $12 \mathrm{~h}$ prior to their study visit. On the morning of their study visit, all participants consumed a standardised breakfast ( 2 pieces of white bread with cheese, tub of plain yoghurt and water), approximately $2 \mathrm{~h}$ prior to their visit. Participants then underwent a baseline FMD assessment of their

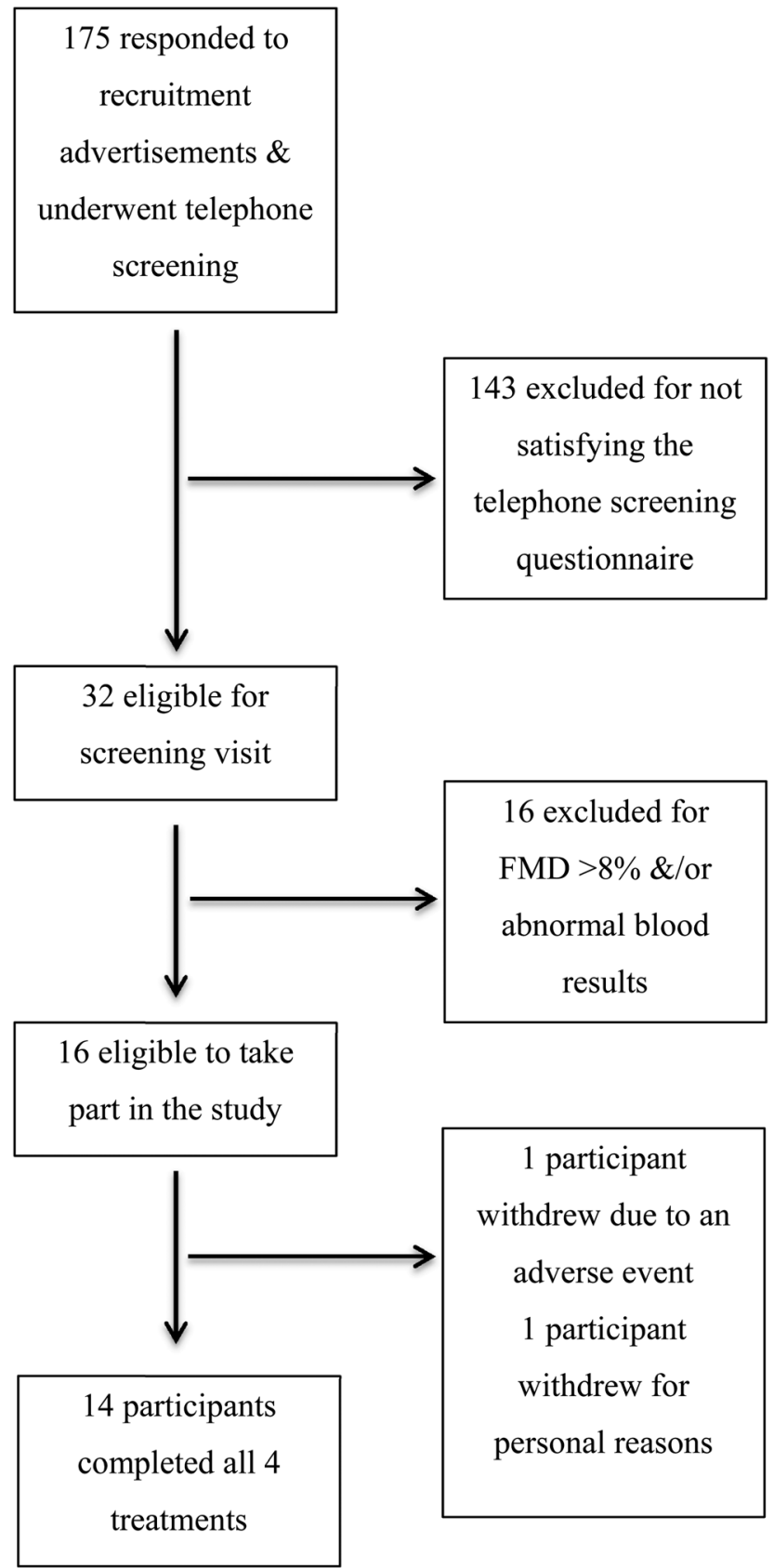

Fig. 1 Study recruitment and enrolment flow chart.

brachial artery, BP measurement and provided a blood sample. They were allocated to receive the study treatments in random order via computer generated block randomisation. Both the participants and the study personnel were blinded to the treatments for the duration of the study and subsequent analysis. Treatments were as follows; (i) $1 \mathrm{~g}$ maltodextrin (control group), (ii) $450 \mathrm{mg}$ purified 5-CGA $+1 \mathrm{~g}$ maltodextrin, (iii) $900 \mathrm{mg}$ purified 5-CGA + $1 \mathrm{~g}$ maltodextrin, or (iv) $200 \mathrm{mg}$ purified (-)-epicatechin $+1 \mathrm{~g}$ maltodextrin (positive control). Treatments were given dissolved in $200 \mathrm{~mL}$ of warm water and consumed within 10 minutes, with a minimum one-week 
washout between visits. At $1 \mathrm{~h}$ and $4 \mathrm{~h}$ post-treatment, participants underwent another FMD assessment and provided a blood sample. BP was monitored every $30 \mathrm{~min}$ from baseline to $4 \mathrm{~h}$. The study was approved by the University of Western Australia, Human Research Ethics Committee.

\section{Flow mediated dilatation}

Brachial artery vasodilator function was assessed using ultrasound according to a previously published protocol. ${ }^{13}$ A single trained ultrasonographer performed all measurements. Endothelium-dependent vasodilation was assessed as response to forearm ischaemic FMD. Briefly, participants rested in a supine position in a quiet, temperature controlled room $\left(21^{\circ} \mathrm{C}\right.$ to $25{ }^{\circ} \mathrm{C}$ ). The left arm was comfortably rested on a foam support and ECG was monitored continuously. A $12-\mathrm{MHz}$ transducer connected to an Acuson Aspen 128 ultrasound device (Acuson Corporation) was fixed in position with a clamp over the brachial artery 5 to $10 \mathrm{~cm}$ proximal to the anticubital crease. A baseline artery diameter recording was performed for $1 \mathrm{~min}$. A BP cuff was then placed around the left forearm and inflated to $200 \mathrm{mmHg}$ for $5 \mathrm{~min}$ to induce reactive hyperaemia. The brachial artery image was continuously recorded for 4 min post cuff deflation to assess FMD. Images were downloaded for retrospective analysis.

Analysis of scans was performed with semi-automated edge-detection software. ${ }^{14,15}$ This automatically calculated the brachial artery diameter, corresponding to the internal diameter. This was gated to the R wave of ECG, with measurements taken at end diastole. Analysis was performed by an experienced observer, blinded to the study treatments. Reproducibility studies have previously demonstrated an intra subject coefficient of variation of $14.7 \%{ }^{15}$ FMD response was assessed in two ways. Firstly, as a peak FMD response following ischaemia. Secondary analysis was carried out by measuring FMD response every 10 seconds from $60 \mathrm{~s}$ (average time to peak dilatation post-ischemia), to $4 \mathrm{~min}$ (240 s) post cuff deflation as a measure of continuous post-ischaemic FMD response. This outlined us the opportunity to detect smaller differences following the CGA treatments. The FMD response provides a guide to NO-mediated endothelial cell function. ${ }^{16}$

\section{Blood pressure assessment}

Clinic blood pressure was monitored every 30 min using a Dinamap 1846SX/P oscillometric recorder (Critikon, Tampa, FL, USA). Subjects were rested for $3 \mathrm{~min}$ in a sitting position. A cuff was fitted to the right arm and 3 readings were taken, $1 \mathrm{~min}$ apart. The first reading was discarded and the second and third averaged to give a final BP. The BP data were analysed at each of the time points and as an overall $4 \mathrm{~h}$ difference between treatment groups.

\section{Plasma nitrite concentrations}

Plasma nitrite concentration, as a reflection of overall nitric oxide status, was measured on freshly isolated plasma using gas phase chemiluminescence. Briefly, blood was collected into $N$-ethylmaleimide (10 mmol $\left.\mathrm{L}^{-1}\right)$ and EDTA (2 $\left.\mathrm{mM}\right)$, mixed and centrifuged immediately at $3000 \mathrm{rpm}$, for $5 \mathrm{~min}$ at $4{ }^{\circ} \mathrm{C}$. Plasma was isolated, kept on ice in the dark and analysed within $1 \mathrm{~h}$ of collection. For determination of nitroso species, fresh plasma $(300 \mu \mathrm{l})$ was treated with sulphanilamide solution $(30 \mu \mathrm{l}, 0.5 \%$ in $0.1 \mathrm{M} \mathrm{HCl}$ ) for $3 \mathrm{~min}$ to remove the endogenous nitrite. Antifoam $(200 \mu \mathrm{l})$ was added prior to injection into the radical purger containing potassium iodide $(0.125 \mathrm{~g})$ and iodine $(0.05 \mathrm{~g})$ in water $(2.5 \mathrm{~mL}) /$ glacial acetic acid $(7.5 \mathrm{~mL})$ at room temperature. Plasma S-nitrosothiols (+nitrosylated species) are quantified by the NO signal peak area of samples pre-treated with sulphanilamide against a nitrite standard $\left(300 \mu \mathrm{l}, 0.5 \mu \mathrm{M} \mathrm{NaNO}{ }_{2}^{-}\right.$). Quantification of NO released by the redox reactions occurs by its chemiluminescence reaction with ozone using a Nitric Oxide Analyser (CLD66, Eco Physics, Sweden).

\section{Plasma CGA concentrations}

Plasma polyphenol concentration was measured on stored plasma samples by liquid chromatography mass spectrometry, at the Nestle Research Centre following completion of the study. Briefly, to $380 \mu \mathrm{l}$ of plasma, $20 \mu \mathrm{l}$ of internal standard (3-(4-hydroxyphenyl)-propionic acid) was added, mixed and placed at $4{ }^{\circ} \mathrm{C}$. The sample was then injected into the Transcend turbulent flow chromatography system (Thermo Fisher Scientific) coupled to a 3200 Q TRAP mass spectrometer instrument equipped with a Turbo Ion Spray ionisation source (Applied Biosystems, Foster City, USA). Blank samples spiked with known concentrations of standard were used to quantify plasma concentrations. ${ }^{17}$

\section{Statistical analysis}

Statistical analysis was carried out using IBM SPSS Statistics Version 21 (2012, Armonk, NY: IBM Corp) and STATA (version 13 StataCorp LP, College Station, TX). All analysis was performed with intention to treat. That is, all participants randomised to the study were included in the final analysis, regardless of whether they completed all four study treatments or followed the recommended diet. Each analysis was performed using a mixed effects regression model to account for the cross-over design with repeated measurements both within and across visits. The model for FMD included fixed effect terms for treatment, visit $(1,2,3$ or 4$)$, scan (baseline, 1 hour or 4 hours), time point ( 60 to 240 seconds in categories of 10 seconds), as well as an interaction term between treatment and scan. Since there was a significant interaction between treatment and scan, we stratified the remaining analysis by scan using the same model for each scan but excluding the scan $\mathrm{X}$ treatment interaction term. The participant ID number was included as a random intercept in each model. The $p$ values for the estimated treatment effects were Bonferroni adjusted to account for the 6 multiple comparisons between the 4 treatments. Mean estimated treatment effects between active treatments and the negative control were obtained using the estimated marginal means from the mixed effects model. 


\section{Results}

\section{Study participant characteristics}

In total, 16 men and women were randomised into the study with 14 participants completing all four treatments. Their baseline characteristics are presented in Table 1 . The mean age was 60 years (range: 39-69 years) and the mean screening FMD was $5.4 \%( \pm 1.2 \%)$.

\section{Effects of 5-CGA on peak FMD response and continuous FMD response}

There was no significant effect on peak FMD response for either dose of 5-CGA or the epicatechin at $1 \mathrm{~h}$ or $4 \mathrm{~h}$ post-treatment (Table 2). Secondary analysis of continuous mean postischaemic FMD response following CGA treatment revealed there was a significant treatment-by-scan interaction $(\mathrm{p}<0.001)$, indicating a difference in FMD response according to treatment over the three scans. At $1 \mathrm{~h}$ post treatment, there was a dose-related effect of 5-CGA to increase continuous FMD response (Fig. 2b). Relative to control, both $450 \mathrm{mg}$ 5-CGA and $900 \mathrm{mg}$ of 5-CGA resulted in a higher continuous FMD response $(0.47 \pm 0.16 \%, p=0.016 ; 0.65 \pm 0.16 \%, p<0.001$; respectively) at $1 \mathrm{~h}$. At $4 \mathrm{~h}$, the only significant difference observed was a higher continuous FMD response with $900 \mathrm{mg}$ 5 -CGA relative to control $(0.44 \pm 0.16 \%, p<0.05)$. There was no significant effect of $450 \mathrm{mg}$ CGA on continuous FMD response $(0.18 \pm 0.17 \%, p=0.99)$ (Fig. 2c). There was no significant

Table 1 Study participant characteristics

\begin{tabular}{ll}
\hline & All participants $(N=16)$ \\
\hline Age (years) & $59.9(8.2)$ \\
Gender & 6 men/10 women \\
Caucasian & 16 \\
Screening FMD (\%) & $5.4(1.2)$ \\
Height $(\mathrm{cm})$ & $168.4(10.0)$ \\
Weight (kg) & $70.5(13.2)$ \\
BMI $\left(\mathrm{kg} \mathrm{m} \mathrm{m}^{-2}\right)$ & $24.7(3.3)$ \\
Systolic BP $(\mathrm{mmHg})$ & $115.1(9.1)$ \\
Diastolic BP $(\mathrm{mmHg})$ & $67.8(7.4)$ \\
Heart rate (bpm) & $56.8(7.4)$
\end{tabular}

Data are presented as mean (SD). effect of epicatechin on mean continuous FMD response (data not shown).

\section{Effects of 5-CGA on blood pressure}

Blood pressure was measured every $30 \mathrm{~min}$ from $0 \mathrm{~h}$ until $4 \mathrm{~h}$. At each time point, there was no significant difference between the $450 \mathrm{mg}$ and $900 \mathrm{mg}$ 5-CGA groups and control, for systolic BP or diastolic BP (Table 3). There was no effect of the $200 \mathrm{mg}$ epicatechin treatment on BP. There was a significant increase in BP across time, across all four treatments combined.

\section{Effects of 5-CGA on plasma nitrite levels}

Circulating plasma nitrite levels were measured as an index of overall NO production. Relative to control, there was no significant effect of $450 \mathrm{mg}$ and $900 \mathrm{mg}$ 5-CGA or $200 \mathrm{mg}$ epicatechin onplasma nitrite concentrations (Table 4).

\section{Circulating plasma CGA metabolites}

The circulating plasma CGA metabolites were measured at baseline and at $1 \mathrm{~h}$ and $4 \mathrm{~h}$ to confirm both compliance and absorbance of the compounds. There were significant increases in CGA metabolites following their respective treatments at both $1 \mathrm{~h}$ and $4 \mathrm{~h}$. Analysis of the individual CGA metabolites reveals the pattern of metabolism over the $4 \mathrm{~h}$ treatment period and the total phenolic acid profile over the $4 \mathrm{~h}$ treatment period (Fig. 3).

\section{Discussion}

The present study found no significant effect of 5-CGA on endothelial function as measured by peak FMD response. However, we did find a statistically significant dose-related effect of 5-CGA on continuous mean FMD response. At $1 \mathrm{~h}$, this improvement was observed for both the $450 \mathrm{mg}$ and $900 \mathrm{mg}$ dose, and this increase was sustained at $4 \mathrm{~h}$ for the $900 \mathrm{mg}$ dose. Epicatechin, a dietary flavonoid found in cocoa and used as a positive control, had no significant effect on FMD, either measured as peak or continuous response. None of the polyphenol treatments had any effect on BP.

There is very little work investigating the direct effect of 5-CGA, the major coffee polyphenol, on vascular function. A recent study investigating the acute effect of $400 \mathrm{mg}$ of 3-CGA

Table 2 Estimated effects of 5-CGA on peak FMD response at 1 hour and 4 hours ${ }^{a}$

\begin{tabular}{|c|c|c|c|c|c|}
\hline & \multicolumn{3}{|l|}{ Time } & \multicolumn{2}{|c|}{$\begin{array}{l}\text { Estimated difference } \\
(95 \% \text { CI) v control }\end{array}$} \\
\hline & $0 \mathrm{~h}$ & $1 \mathrm{~h}$ & $4 \mathrm{~h}$ & $1 \mathrm{~h}$ & $4 \mathrm{~h}$ \\
\hline Control $(n=14)$ & $6.7(2.5)$ & $7.1(3.0)$ & $7.6(2.5)$ & & \\
\hline $450 \mathrm{mg}$ 5-CGA $(n=15)$ & $6.5(2.1)$ & $7.1(2.3)$ & $6.9(3.1)$ & $0.78(-0.96,2.52)$ & $-0.06(-1.82,1.71)$ \\
\hline $900 \mathrm{mg} 5-\mathrm{CGA}(n=14)$ & $6.1(1.8)$ & $7.2(2.9)$ & $7.2(2.6)$ & $0.71(-1.02,2.43)$ & $0.22(-1.55,1.99)$ \\
\hline Epicatechin $(n=15)$ & $6.1(1.2)$ & $6.8(2.0)$ & $7.5(2.8)$ & & \\
\hline
\end{tabular}

Results are presented as mean (SD). ${ }^{a}$ Estimated differences at 1 hour and 4 hours were obtained from a mixed effects regression model (see statistical methods section for details). 
(a) Baseline FMD response. No significant differences between groups.

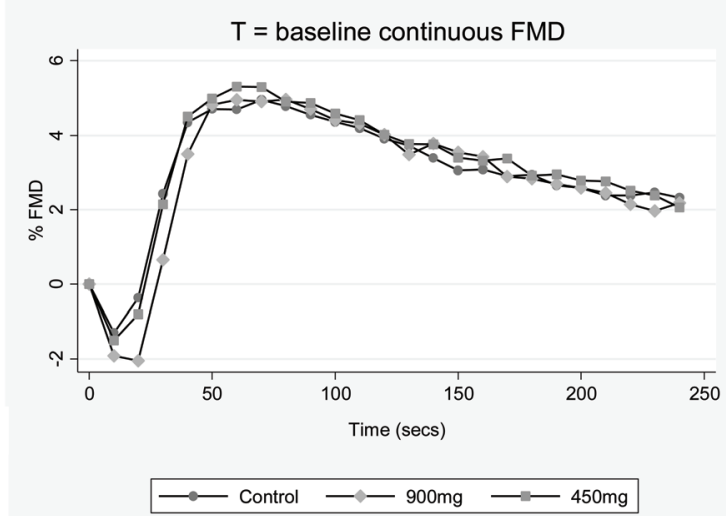

(b) FMD response at $1 \mathrm{hr}$. Both $450 \mathrm{mg} 5$-CGA and $900 \mathrm{mg}$ of 5-CGA resulted in a higher FMD response $(0.47 \pm 0.16 \%, \mathrm{p}=0.016 ; 0.65 \pm 0.16 \%, \mathrm{p}<0.001$; respectively).

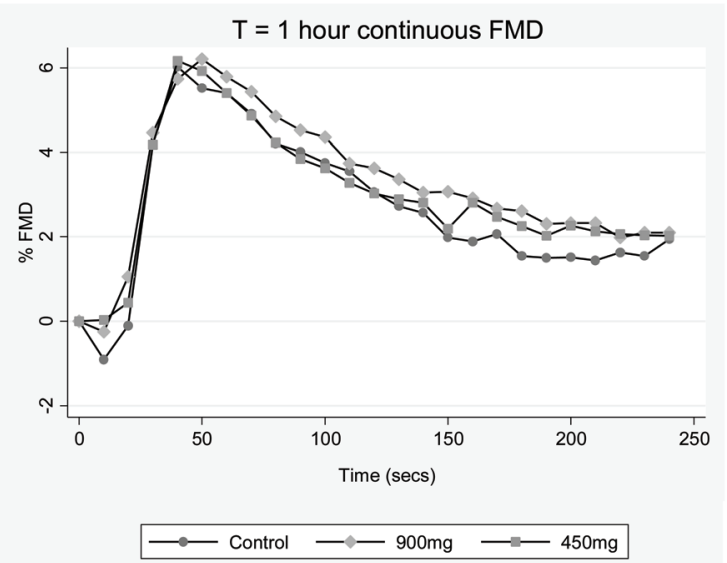

(c) At 4 hrs, $900 \mathrm{mg} 5$-CGA was higher than control $(0.44 \pm 0.16 \%$, p $<0.05)$ but there was no significant effect of $450 \mathrm{mg}$ CGA $(0.18 \pm 0.17 \%$, $\mathrm{p}=0.99)$

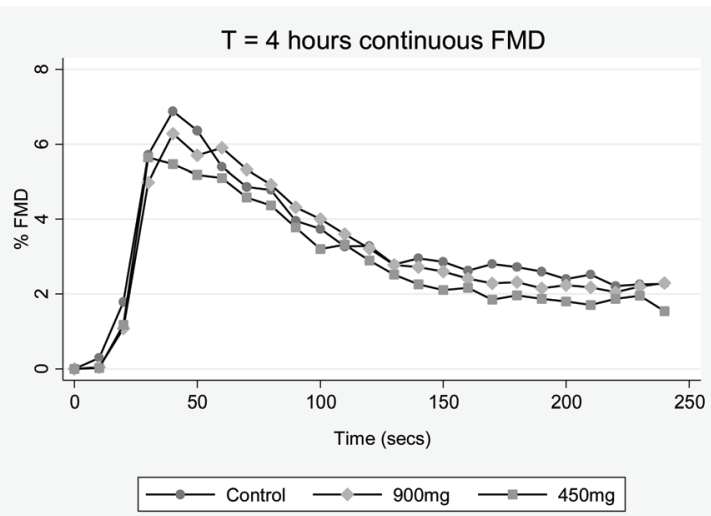

Fig. 2 Observed FMD response between treatments from 0 seconds to 240 seconds post cuff deflation at (a) baseline (b) $1 \mathrm{~h}$ post-treatment, and (c) $4 \mathrm{~h}$ post-treatment. Estimated effects were obtained using mixed models with data for 60-240 seconds post cuff-deflation.

in healthy subjects demonstrated a reduction in BP at $1 \mathrm{~h}$ post consumption. However, this was not associated with any improvement in peak FMD response or circulating nitroso/ nitric oxide species..$^{10}$ In the present study, we used a comparable dose of 5-CGA, as well as a higher dose, and measured BP every 30 minutes and FMD response at both $1 \mathrm{~h}$ and $4 \mathrm{~h}$ postconsumption. Although we did not observe any improvement in BP or peak FMD response, there was a significant doserelated effect on continuous post-ischaemic FMD response. Measurement of the continuous FMD dilatory response provides greater statistical power and allows us to observe smaller, yet still statistically significant, results. It may also be more physiologically relevant than measurement of a single time point peak response, representing a sustained improvement on vascular function.

The low dose used in the present study corresponds to the amount of CGA found in approximately 4 cups of regular roasted coffee (or 2 cups of a CGA-enriched coffee), while the high dose corresponds to approximately 8 cups of regular coffee (or 4 cups of a CGA-enriched coffee). However, the exact amount of CGA found in a regular cup of coffee can differ depending on both the type of coffee used and the brewing methods. ${ }^{18}$ Following ingestion, CGA undergoes extensive metabolic transformation in both the intestine and colon before absorption, as well as following absorption in the liver. As a result, it may be the metabolites of CGA (and other ingested polyphenols) that have significant biological activity and subsequent health benefits. ${ }^{2}$ Although the bioavailability of these metabolites has been studied, it is likely that this is also affected by biological and chemical differences, as well as individual differences in enzymatic modification, metabolite transport and gut microflora. ${ }^{2,4}$ Although participants were advised to reduce their polyphenolic intake prior to each study visit, we cannot rule out the influence of background diet on both the metabolism of these compounds and their subsequent in vivo effects. As a result, there may be significantly different responses and effects between individuals following both coffee consumption and ingestion of isolated pure polyphenolic compounds. The modest increase in continuous mean FMD response that we observed at $1 \mathrm{~h}$ may reflect peak plasma CGA or metabolite concentrations following transformation of the parent compound. This effect was diminished at $4 \mathrm{~h}$ post consumption at the low dose, suggesting the active metabolites were either excreted, or further metabolised into inactive compounds.

It is somewhat surprising that epicatechin, our positive control, had no effect on peak FMD response, particularly given several other studies have previously demonstrated a benefit. ${ }^{8,9}$ The dose used in the present study was similar to that used in previous studies, and while Loke et al. didn't measure FMD response directly, Schroeter et al. measured response at $2 \mathrm{~h}$ post-treatment, although it should be noted that this was only performed in 3 study participants. It may be that by measuring FMD response at $1 \mathrm{~h}$ and $4 \mathrm{~h}$ in the present study, we have missed the peak beneficial effect of this particular dietary polyphenol on endothelial function.

There are a number of limitations to consider when interpreting the results of the present study. Although the study was a randomised controlled cross-over study, the number of participants was small, with 14 completing all four treatment arms. This limited our power to detect a relatively small 
Table 3 Effects of 5-CGA on blood pressure

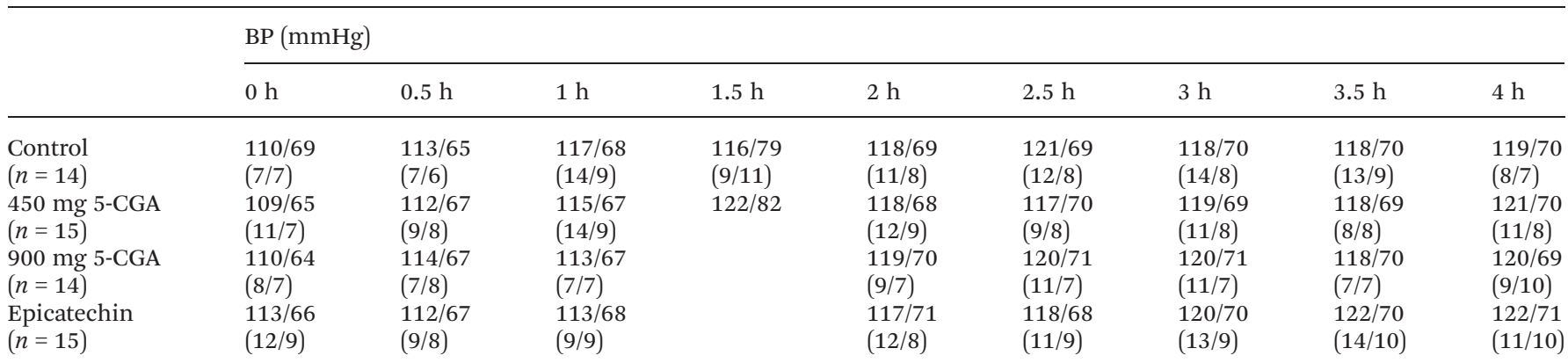

Results are presented as mean systolic BP/mean diastolic BP (SD).

Table 4 Effects of 5-CGA on plasma nitrite at 1 hour and 4 hours

\begin{tabular}{llll}
\hline & \multicolumn{3}{l}{ Nitrate $(\mathrm{nM})$} \\
\cline { 2 - 4 } & $0 \mathrm{~h}$ & $1 \mathrm{~h}$ & $4 \mathrm{~h}$ \\
\hline Control $(n=14)$ & $131(98)$ & $237(229)$ & $205(97)$ \\
$450 \mathrm{mg} 5-C G A(n=15)$ & $123(72)$ & $187(73)$ & $218(87)$ \\
$900 \mathrm{mg}$ 5-CGA $(n=14)$ & $123(62)$ & $195(125)$ & $185(106)$ \\
Epicatechin $(n=15)$ & $164(203)$ & $250(258)$ & $165(78)$ \\
& & &
\end{tabular}

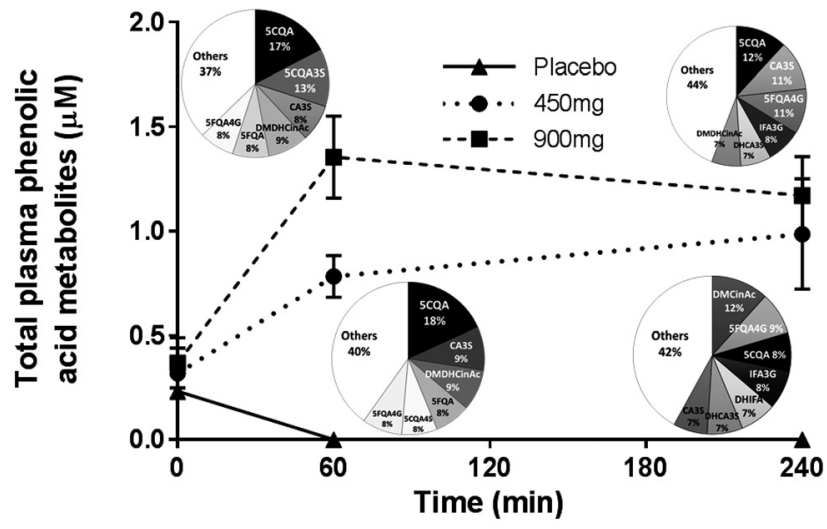

Fig. 3 Plasma distribution of CGA metabolites at baseline, $1 \mathrm{~h}$ and $4 \mathrm{~h}$ post-treatment.

change in FMD response. Secondly, it might be that important time points have been missed in choosing when to measure FMD response. This is particularly noticeable when considering the lack of effect seen with epicatechin. The time points chosen for this study were based on peak times for CGA and its metabolites. Thirdly, although we recruited participants based a screening FMD response between 3-8\%, they were considered to be otherwise healthy. This may mean that any observed benefit of the CGA compounds may have been too small to detect, as the participants weren't considered to be a high-risk group with endothelial dysfunction. Future studies in populations with existing CVD or pre-existing CVD risk factors may help to elucidate the beneficial effects of this com- pound on vascular function. Furthermore, the use of an isolated compound, specifically 5-CGA, may have also limited the interpretation of our findings. Although it is considered one of the major polyphenolic compounds in coffee, there are many other compounds present. Use of a single, purified compound in the present study may have meant that any additive or synergistic effects of other polyphenols also found in coffee (particularly after roasting), could not be observed.

In conclusion, there was no significant effect of 5-CGA or epicatechin on the peak FMD response, our primary outcome. We did observe a dose-related improvement in continuous mean FMD response for 5-CGA at $1 \mathrm{~h}$ post ingestion, which was diminished by $4 \mathrm{~h}$. Future studies examining whole coffee, as well as high-risk population groups, are warranted.

\section{Acknowledgements}

The authors acknowledge the assistance of Lisa Rich in the performance and analysis of all FMD measurements and Adeline Hoeneveld for the analysis of plasma nitrite concentrations. The study was funded by Nestle (NRC 11.35). NCW acknowledges the previous support of a RPH Medical Research Foundation/UWA Postdoctoral Fellowship. JMH acknowledges the support of a NHMRC Research Fellowship.

\section{References}

1 L. A. Bazzano, J. He, L. G. Ogden, C. M. Loria, S. Vupputuri, L. Myers, et al., Fruit and vegetable intake and risk of cardiovascular disease in US adults: the first National Health and Nutrition Examination Survey Epidemiologic Follow-up Study, Am. J. Clin. Nutr., 2002, 76(1), 93-99.

2 S. K. Bohn, N. C. Ward, J. M. Hodgson and K. D. Croft, Effects of tea and coffee on cardiovascular disease risk, Food Funct., 2012, 3(6), 575-591.

3 Y. Zhao, J. Wang, O. Ballevre, H. Luo and W. Zhang, Antihypertensive effects and mechanisms of chlorogenic acids, Hypertens. Res., 2012, 35(4), 370-374. 
4 I. A. Ludwig, M. N. Clifford, M. E. Lean, H. Ashihara and A. Crozier, Coffee: biochemistry and potential impact on health, Food Funct., 2014, 5(8), 1695-1717.

5 R. Huxley, C. M. Lee, F. Barzi, L. Timmermeister, S. Czernichow, V. Perkovic, et al., Coffee, decaffeinated coffee, and tea consumption in relation to incident type 2 diabetes mellitus: a systematic review with meta-analysis, Arch. Intern. Med., 2009, 169(22), 2053-2063.

6 J. N. Wu, S. C. Ho, C. Zhou, W. H. Ling, W. Q. Chen, C. L. Wang, et al., Coffee consumption and risk of coronary heart diseases: a meta-analysis of 21 prospective cohort studies, Int. J. Cardiol., 2009, 137(3), 216-225.

7 S. C. Larsson and N. Orsini, Coffee consumption and risk of stroke: a dose-response meta-analysis of prospective studies, Am. J. Epidemiol., 2011, 174(9), 993-1001.

8 W. M. Loke, J. M. Hodgson, J. M. Proudfoot, A. J. McKinley, I. B. Puddey and K. D. Croft, Pure dietary flavonoids quercetin and (-)-epicatechin augment nitric oxide products and reduce endothelin-1 acutely in healthy men, Am. J. Clin. Nutr., 2008, 88(4), 1018-1025.

9 H. Schroeter, C. Heiss, J. Balzer, P. Kleinbongard, C. L. Keen, N. K. Hollenberg, et al., (-)-Epicatechin mediates beneficial effects of flavanol-rich cocoa on vascular function in humans, Proc. Natl. Acad. Sci. U. S. A., 2006, 103(4), 1024-1029.

10 A. Mubarak, C. P. Bondonno, A. H. Liu, M. J. Considine, L. Rich, E. Mas, et al., Acute effects of chlorogenic acid on nitric oxide status, endothelial function, and blood pressure in healthy volunteers: a randomized trial, J. Agric. Food Chem., 2012, 60(36), 9130-9136.

11 A. Stalmach, W. Mullen, D. Barron, K. Uchida, T. Yokota, C. Cavin, et al., Metabolite profiling of hydroxycinnamate derivatives in plasma and urine after the ingestion of coffee by humans: identification of biomarkers of coffee consumption, Drug Metab. Dispos., 2009, 37(8), 1749-1758.

12 T. W. Crozier, A. Stalmach, M. E. Lean and A. Crozier, Espresso coffees, caffeine and chlorogenic acid intake: potential health implications, Food Funct., 2012, 3(1), 3033.

13 N. C. Ward, K. D. Croft, J. Hodgson, L. Rich, L. J. Beilin and I. B. Puddey, Brachial artery vasomotor function is inversely associated with 24-h ambulatory blood pressure, J. Hypertens., 2004, 22(5), 967-972.

14 G. Dogra, L. Rich, K. Stanton and G. F. Watts, Endothelium-dependent and independent vasodilation studies at normoglycaemia in type I diabetes mellitus with and without microalbuminuria, Diabetologia, 2001, 44(5), 593601.

15 R. J. Woodman, D. A. Playford, G. F. Watts, C. Cheetham, C. Reed, R. R. Taylor, et al., Improved analysis of brachial artery ultrasound using a novel edge-detection software system, J. Appl. Physiol., 2001, 91(2), 929-937.

16 A. J. Flammer, T. Anderson, D. S. Celermajer, M. A. Creager, J. Deanfield, P. Ganz, et al., The assessment of endothelial function: from research into clinical practice, Circulation, 2012, 126(6), 753-767.

17 C. Marmet, L. Actis-Goretta, M. Renouf and F. Giuffrida, Quantification of phenolic acids and their methylates, glucuronides, sulfates and lactones metabolites in human plasma by LC-MS/MS after oral ingestion of soluble coffee, J. Pharm. Biomed. Anal., 2014, 88, 617-625.

18 I. A. Ludwig, P. Mena, L. Calani, C. Cid, D. Del Rio, M. E. Lean, et al., Variations in caffeine and chlorogenic acid contents of coffees: what are we drinking?, Food Funct., 2014, 5(8), 1718-1726. 